\title{
INTERESES AMBIENTALES PRIORITARIOS DE LA POBLACIÓN EN SITUACIÓN DE POBREZA EXTREMA. ESTUDIO DE CASO: BARRIO EL ENSUEÑO, CÓRDOBA, QUINDÍO.
}

\author{
Paula Andrea López Arbeláez ${ }^{1}$ \\ Recibido el 23 de julio de 2015, aprobado el 26 de octubre de 2015 y actualizado el \\ 19 de mayo de 2016
}

DOI: 10.17151/luaz.2016.43.11

\section{RESUMEN}

El presente artículo analiza los intereses ambientales prioritarios de la población en situación de pobreza extrema del barrio El Ensueño, del municipio de Córdoba, y de las instituciones públicas y privadas que atienden esta población a través de sus programas para comprender los aspectos menos evidentes, como lo son el surgimiento de conflictos ambientales. Dada la complejidad del estudio se utilizó un enfoque metodológico de análisis-síntesis para dar cuenta de una investigación analítica, utilizando métodos cualitativos. Los conflictos entre las familias en situación de pobreza extrema y las entidades públicas y privadas seleccionadas para el estudio de caso obedecen a la debilidad institucional, que se traduce en el mínimo impacto que tienen los programas sociales y el alto nivel de centralismo en el cual los beneficiarios de los programas son seleccionados desde el nivel nacional obedeciendo más a criterios políticos que técnicos. Este hecho genera conflictos, no solo entre las entidades y las familias directamente implicadas, sino también entre las mismas entidades del Estado. En ese orden de ideas, los intereses ambientales prioritarios pueden ser un instrumento desde las ciencias ambientales para aportar a la evaluación de las políticas públicas en Colombia ya que permiten unificar variables económicas, sociales y ambientales a través de la visión integral de una problemática ambiental. De igual forma, en el escenario de implementación de un posible acuerdo de paz con la guerrilla de las FARC, en el que se prevé la necesidad de diseñar e implementar políticas públicas en construcción de paz para conciliar los conflictos sociales y ambientales de los territorios más golpeados por el conflicto armado, abordar los intereses ambientales prioritarios permitiría la identificación y triangulación de información social y ambiental para la resolución de estos. 


\section{PALABRAS CLAVE}

Pobreza extrema, ambiente, intereses ambientales prioritarios, conflictos.

\section{PRIORITY ENVIRONMENTAL INTERESTS OF THE POPULATION IN SITUATION OF EXTREME POVERTY. CASE STUDY: BARRIO EL ENSUEÑO, CORDOBA, QUINDÍO}

This article discusses the priority environmental interests of the population in extreme poverty of the neighborhood El Ensueño, municipality of Cordoba, and of the public and private institutions that serve this population through their programs to understand the aspects less evident, as are the emergence of environmental conflicts. Given the complexity of the study used a methodological approach to analysis-synthesis to give an account of an analytical research, using qualitative methods. Conflicts between families in extreme poverty and the public and private institutions selected for the case study are due to the institutional weakness, that translates into minimal impact that have the social programs and the high level of centralism in which the beneficiaries of the programs are selected from the national level in obedience to political criteria more than technical ones. This fact gives rise to conflict, not only between the entities and their families directly involved, but also between the same entities of the State. In that order of ideas, the priority environmental interests can be an instrument from the environmental sciences to contribute to the evaluation of public policies in Colombia, because they allow you to consolidate economic variables, social environmental and through the holistic view of environmental problems. Similarly, in the deployment scenario of a possible peace agreement with the guerrillas of the FARC, which provides for the need to design and implement public policies in peace-building to reconcile the social and environmental conflicts of the territories hit hardest by the armed conflict, address environmental concerns priority would enable the identification and triangulation of social and environmental information for the resolution of these.

\section{KEY WORDS}

Extreme poverty, environment, priority environmental interests, conflicts. 


\section{INTRODUCCIÓN}

La investigación nace de la inquietud de entender la manera como la mayoría de estudios abordan la problemática ambiental asociada a la pobreza, y de cómo estos guían muchas veces la política en materia de gestión ambiental para la "sostenibilidad", sin tener en cuenta los intereses de los directamente afectados que son las personas en situación de pobreza extrema. En relación al anterior punto, el Programa de las Naciones Unidas para el Desarrollo -PNUD- establece:

Las personas y los grupos afectados más adversamente son quienes están en peor situación y menos empoderados. Las prioridades en materia de políticas pueden no reflejar sus intereses y necesidades. En muchos países y contextos, las desigualdades de poder afectan los resultados ambientales, que son mediados por las instituciones políticas y sociales. (PNUD, 2011, p. 72)

El estudio de caso del barrio El Ensueño, en el municipio de Córdoba, es el escenario seleccionado para el trabajo de investigación, no solo por los problemas ambientales puntuales que se manifiestan y que son producto de las interacciones históricas entre los subsistemas natural, humano y construido, sino también por su localización en una zona periurbana, común en municipios pequeños, alejados de grandes centros urbanos, donde sus pobladores combinan el trabajo en el campo con las comodidades y facilidades de la vida urbana.

Tomando una línea de tiempo desde 1990 hasta diciembre de 2013, se analizan las diferentes situaciones que han configurado la problemática ambiental actual que afecta a las familias en situación de pobreza extrema del barrio El Ensueño, cómo esta problemática ha afectado sus capacidades generando carencias en un círculo vicioso configurando trampas de pobreza y conflictos ambientales y socioambientales debido a la incompatibilidad con los intereses ambientales prioritarios de las entidades públicas y privadas, cuya misión es generar capacidades en la población.

Sobre las relaciones pobreza-ambiente, en el marco teórico se desarrollan algunas ideas comúnmente conocidas sobre la degradación ambiental que la pobreza suele producir o viceversa. Desde la perspectiva de Sen (citado en Corredor, 1998, p. 60) se explica cómo la degradación del ambiente limita las capacidades de las personas para llevar la vida que valoran, limitando sus libertades de elegir el ser y el hacer. También, se exploran algunas ideas de otros autores sobre cómo la 
desigualdad o distribución social de los elementos del ambiente limitan las capacidades de las personas viéndose forzadas a intensificar la presión sobre los elementos del medio natural, generando o incrementando la degradación ambiental, la cual, a su vez, intensifica la pobreza porque limita las capacidades de las personas, en un círculo vicioso.

Los Intereses Ambientales Prioritarios, tema central del trabajo de investigación, tanto de la población en situación de pobreza extrema, como de las instituciones que tienen a cargo atender y solucionar los problemas de la población en general, se abordan desde los postulados que ofrecen la teoría de conflictos ambientales de Fontaine (2004, p. 520), la teoría del desarrollo libertario de Sen (citado en Corredor, 1998, p. 60), la teoría de la ecología social de Gudynas \& Evia (1991, p. 26) y desde distintas perspectivas como la filosofía y el derecho expuestas por Posada (2009).

La investigación se planteó como objetivos: El diagnóstico de la problemática ambiental que afecta a la población en situación de pobreza extrema del barrio El Ensueño. La identificación de los intereses ambientales prioritarios de la población en situación de pobreza extrema del barrio El Ensueño y de las instituciones públicas. Y la determinación de la existencia de conflictos ambientales entre los intereses ambientales prioritarios de la población en situación de pobreza extrema del barrio El Ensueño y la institucionalidad pública del municipio de Córdoba, Quindío.

Para las ciencias ambientales, el estudio es importante porque ayuda a precisar y articular los marcos teóricos y metodológicos en los estudios que triangulen desarrollo, pobreza, ambiente, interés ambiental prioritario y conflicto ambiental en comunidades de extrema pobreza y aportará a la interpretación en territorios locales de las interdependencias de las relaciones de desigualdad social, degradación ambiental y conflictos ambientales.

\section{MATERIALES Y MÉTODO}

La investigación se presenta como un estudio analítico situacional (Hurtado, 2000, p. 279) ya que se ausculta de forma intensiva la problemática ambiental de las familias seleccionadas para el caso de estudio, permitiendo una comprensión más profunda de su situación de vulnerabilidad, estableciendo las relaciones existentes 
entre las carencias de los bienes tangibles e intangibles con la profundización de la pobreza extrema.

La investigación analítica incluye tanto el análisis como la síntesis, esto quiere decir que si en el análisis se desintegran las partes del todo para su comprensión, en el proceso de síntesis se reúnen varias cosas de modo que conformen una totalidad diferente (Hurtado, 2000, p. 269). En ese orden de ideas, la investigación partió de la problemática ambiental para identificar los intereses ambientales prioritarios de la población en situación de pobreza extrema y de las entidades públicas encontrando los conflictos entre ambas partes.

Con el fin de responder a las complejidades propias de este estudio, se incorporaron métodos cualitativos que permitieron una comprensión de la problemática ambiental que afecta a la población en situación de pobreza extrema del barrio El Ensueño del municipio de Córdoba. Metodológicamente se asume que las interacciones históricas entre el sistema ambiental (conformado por el subsistema natural, humano y construido) y el sistema humano (conformado por las familias en situación de pobreza extrema del barrio El Ensueño seleccionadas para el estudio de caso) han dado origen a los problemas ambientales que configuran la problemática ambiental.

En la Figura 1 se representa el modelo metodológico utilizado desde la teoría de la ecología social, donde la problemática ambiental se configura cuando el sistema ambiental interactúa con el sistema humano. De la problemática ambiental surgen los intereses ambientales prioritarios, tanto de las familias en situación de pobreza extrema como de las entidades públicas y privadas. Las diferentes posiciones frente a estos intereses dan origen a su vez a los conflictos ambientales. 

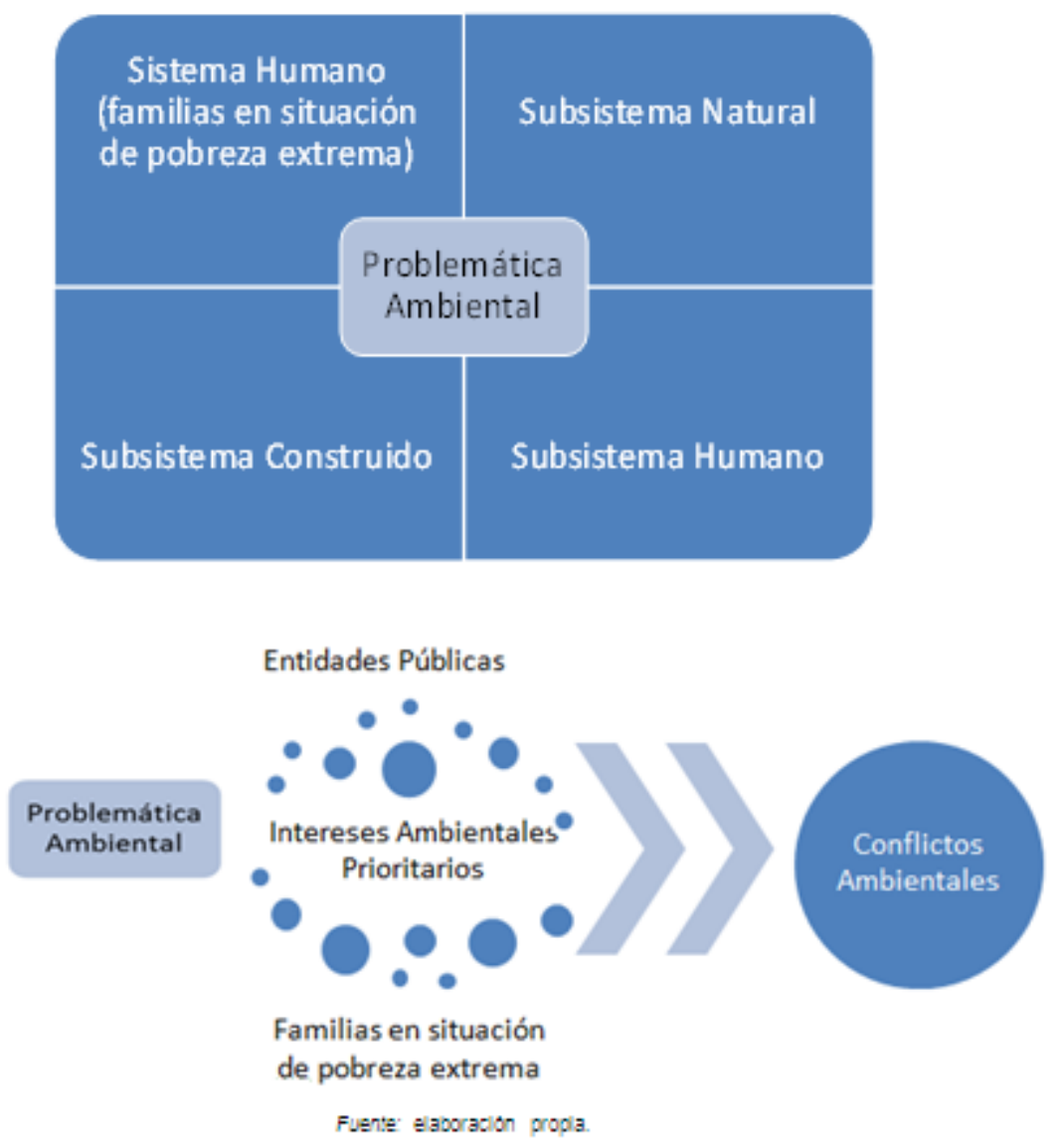

Figura 1. Modelo metodológico.

El trabajo de investigación se llevó a cabo en tres fases: la fase explorativa, la fase interactiva y la fase de análisis y discusión de resultados. A continuación se explica el desarrollo de cada una de ellas.

\section{Fase explorativa}

Durante esta fase se llevaron a cabo tres actividades que fueron el acercamiento inicial a la población que habita en el barrio El Ensueño, la revisión de información proveniente de fuentes secundarias y la selección de las familias para el estudio de caso.

El acercamiento inicial al barrio se hizo a través de los cogestores sociales de la Estrategia Unidos, quienes han estado prestando sus servicios de 
acompañamiento a 45 familias en situación de pobreza extrema desde el año 2008. La primera actividad que se realizó fue un recorrido por todo el barrio donde los cogestores sociales señalaron las principales manifestaciones de la problemática ambiental, como el riesgo evidente al que están expuestos los habitantes del barrio, los problemas en el manejo de los residuos sólidos, los problemas al interior de algunas viviendas, entre otras. A través de los cogestores sociales también se realizó el acercamiento a la presidenta de la Junta de Acción Comunal del barrio.

La revisión de la información de fuentes secundarias se realizó con el objetivo de reconstruir la historia de la construcción del barrio, así como las diferentes situaciones por las que han tenido que pasar los habitantes del municipio, como fueron la crisis económica de los noventa, el terremoto, la reconstrucción y el conflicto armado, siendo necesario consultar el archivo del FOREC (Fondo para la Reconstrucción y Desarrollo Social del Eje Cafetero) que se encuentra en la Biblioteca de la Universidad del Quindío, los informes de riesgo de la Defensoría del Pueblo y algunos libros sobre el proceso de la reconstrucción del terremoto. Durante la consulta del proceso de reconstrucción del terremoto se tuvo en cuenta el material bibliográfico que las universidades presentaron, ya que el material de las instituciones públicas no presentaba información objetiva.

Para la selección de las familias con las cuales se llevó a cabo el proceso de investigación, la presidenta de la Junta de Acción Comunal ayudó a identificar las familias en situación de pobreza extrema, pertenecientes a la Estrategia Unidos, que cumplieran con alguno de los siguientes criterios: familias cuyos integrantes sean líderes de la comunidad, familias que tengan niños, niñas y adolescentes, familias cuya ubicación geográfica las hace vulnerables a las amenazas del subsistema natural, familias donde la mujer sea cabeza de hogar y familias donde la mujer sea líder de la comunidad.

Las anteriores condiciones para seleccionar las familias como estudio de caso se basaron en los hallazgos del Informe sobre Desarrollo Humano 2011 (PNUD, 2011, p. 8) acerca del mayor riesgo de lesiones o muertes debido a desastres naturales que enfrentan los niños, niñas, mujeres y ancianos de ambos sexos debido a disparidades en la exposición y acceso a recursos, capacidades y oportunidades. En la Tabla 1 se relacionan las familias seleccionadas para la investigación y el criterio de selección. 
Tabla 1. Familias seleccionadas para el estudio de caso

\begin{tabular}{|c|l|}
\hline Núcleo familiar & \multicolumn{1}{|c|}{ Criterio de selección } \\
\hline Núcleo familiar $\mathrm{N}^{0} 1$ & Familia con niños \\
\hline Núcleo familiar $\mathrm{N}^{0} 2$ & Miembro Junta de Acción Comunal \\
\hline Núcleo familiar $N^{0} 3$ & $\begin{array}{l}\text { Familia con adolescentes } \\
\text { Vivienda vulnerable a amenazas de subsistema natural }\end{array}$ \\
\hline Núcleo familiar $\mathrm{N}^{0} 4$ & $\begin{array}{l}\text { Familia con niños } \\
\text { Vivienda vulnerable a amenazas de subsistema natural }\end{array}$ \\
\hline Núcleo familiar $\mathrm{N}^{0} 5$ & $\begin{array}{l}\text { Familia con adolescentes } \\
\text { Vivienda vulnerable a amenazas de subsistema natural }\end{array}$ \\
\hline Núcleo familiar $\mathrm{N}^{0} 6$ & Vivienda vulnerable a amenazas de subsistema natural \\
\hline
\end{tabular}

Fuente: elaboración propia.

\section{Fase interactiva}

En esta fase se identificaron los diferentes elementos, componentes e interrelaciones que configuran la problemática ambiental a través de la recolección de datos por medio de las siguientes técnicas: observación, entrevistas en profundidad, entrevistas estructuradas y mapas parlantes.

\section{Observación}

En la investigación cualitativa la observación constituye otro instrumento adecuado para acceder al conocimiento cultural de los grupos, a partir de registrar las acciones de las personas en su ambiente cotidiano. (...).

Observar con un sentido de indagación científica, implica focalizar la atención de manera intencional, sobre algunos segmentos de la realidad que se estudia, tratando de captar sus elementos constitutivos y la manera cómo interactúan entre sí, con el fin de reconstruir inductivamente la dinámica de la situación. (Bonilla-Castro \& Rodríguez, 1997, p. 118) 
La observación fue una técnica que permitió la recolección de información para convalidarla e integrarla a los demás resultados del trabajo, teniendo en cuenta que fue la problemática ambiental y sus manifestaciones las que determinaron los aspectos observados. La observación se llevó a cabo durante dos jornadas de trabajo de campo en días hábiles, en horas de la tarde. El instrumento utilizado para esta actividad fue un diario de campo.

En la primera jornada de trabajo se realizó un recorrido por todo el casco urbano del municipio donde los cogestores sociales de la Estrategia Unidos relataron la situación de emergencia vivida en el municipio y en el barrio El Ensueño con la ola invernal del año 2010. Durante el recorrido se visitaron los tramos urbanos de las quebradas La Mosca, La Española y La Siberia que causaron la emergencia en el barrio. De igual forma, se hizo un recorrido por el nacimiento de la quebrada La Siberia para verificar los relatos de la presidenta de la Junta de Acción Comunal del barrio El Ensueño sobre el riesgo que representa para los habitantes el abandono de una cantera de material en este lugar.

La segunda jornada de observación se llevó a cabo durante un taller que la Corporación Autónoma Regional del Quindío -CRQ- realizó en el barrio para sensibilizar a los habitantes sobre el tema del manejo de los residuos sólidos. En el diario de campo se consignaron las observaciones realizadas frente a la participación de la comunidad y los aportes que hicieron los asistentes.

\section{Entrevistas en profundidad}

Son una herramienta de diagnóstico cualitativo que se produce a través de encuentros cara a cara con los informantes, para comprender situaciones particulares o experiencias. Lo focalizado se asocia con el hecho de concentrar en un solo punto un conjunto de cosas, conceptos y cuestiones referidas a un tema y a un contenido. (UNIFEM, 2010, p. 74)

El objetivo de realizar las entrevistas en profundidad para las familias seleccionadas era conocer su historia de vida, las distintas situaciones que los llevaron a habitar en el barrio y a seguir habitando en él, las carencias de bienes tangibles e intangibles y cómo estas carencias afectan la calidad de vida de cada uno de los miembros de la familia, además de la relación de estas carencias con las manifestaciones de la problemática ambiental. De igual forma, conocer la percepción de las familias frente a dicha problemática ambiental, qué es más 
importante o a qué le dan más prioridad y qué piensan sobre la gestión de las entidades públicas.

Se hicieron dos entrevistas en profundidad a cada una de las familias que se seleccionaron como estudio de caso. Las entrevistas se realizaron a la persona que la familia identificó como el jefe o jefa de hogar y se llevó a cabo en cada una de sus viviendas. Teniendo en cuenta la recomendación de la presidenta de la Junta de Acción Comunal sobre la disponibilidad de las familias del barrio las entrevistas se hicieron los días sábados en horas de la tarde.

En la primera entrevista se indagó por la historia de vida de las familias, desde el momento en que ocurrió el terremoto hasta la fecha de aplicación del instrumento.

En la segunda entrevista se indagó sobre la situación de la familia frente a los indicadores de los bienes tangibles e intangibles de la Matriz de Pobreza y Vulnerabilidades, como son: alimentación, salud, educación, vivienda, alta dependencia económica, localización en espacios de alto riesgo ambiental, sentido de pertenencia, seguridad, justicia, autonomía y ambiente.

De igual forma, se le preguntó a cada una de las familias seleccionadas por los problemas que consideraban más importantes y urgentes de solucionar, tanto a nivel familiar como del barrio, además de la percepción que tenían frente a la gestión de las entidades públicas.

\section{Entrevistas estructuradas}

Son una herramienta de diagnóstico:

(...) en el que las preguntas se plantean siempre en el mismo orden y se formulan en los mismos términos debido a que su intencionalidad está determinada a razón de un objetivo (...). Este tipo de entrevistas requieren un formato estandarizado y las preguntas deben ser preparadas de antemano a fin de presentar una secuencia predeterminada. (Díaz, 2007, p. 172)

El objetivo de realizar las entrevistas estructuradas a los funcionarios de las entidades públicas y privadas que prestan sus servicios en el municipio de Córdoba era identificar las prioridades de la gestión de estas entidades, 
específicamente los intereses ambientales prioritarios definidos en el marco teórico como una de las categorías analíticas de la investigación.

Las entrevistas se solicitaron a la alta dirección de las entidades, sin embargo, en algunos casos asignaron a un funcionario en representación del Director. Además de entidades públicas también se realizaron entrevistas a algunas entidades privadas que se identificaron durante el trabajo de campo porque estaban realizando intervenciones directamente en el barrio El Ensueño, como es el caso de la Cruz Roja Colombiana Seccional Quindío y de la Fundación Concívica.

\section{Mapas parlantes}

La técnica de los mapas parlantes ha sido ampliamente utilizada en el rescate de los saberes locales sobre todo en los aspectos territoriales y administrativo-políticos. Es muy útil para la sistematización espacial de diversos temas productivos, ambientales, sociales, etc. (...).

La técnica consiste en lograr el dibujo de un mapa del área específica de estudio (...) a partir del trabajo de grupos. Se anima la representación gráfica y literal de los aspectos que consideran más importantes del área de estudio (...): límites y colindancias, zonificación local, recursos hídricos (...), relieve (...), infraestructura (...), etc. (Cox, 1996, p. 19)

Para la elaboración de los mapas parlantes se realizó un taller que contó con la participación de las familias seleccionadas para el estudio, además de otras familias habitantes del barrio (pertenecientes a la Estrategia Unidos). No fue necesaria una fase previa de acercamiento porque las familias ya tenían conocimiento sobre la realización de la investigación y ya habían tenido contacto con la investigadora.

Para la convocatoria se solicitó el apoyo de la presidenta de la Junta de Acción Comunal, quien aprovechó el espacio para socializar otros temas de importancia para los habitantes del barrio. La información de los mapas parlantes se consolidó en una matriz que se presenta en la Tabla 2, en la cual se identificó la carencia con la que se relacionaban los problemas identificados por las familias, la importancia que estas daban a dichos problemas, la identificación de los agentes que causaban la manifestación del problema, las causas y la posible solución. 
Tabla 2. Matriz de identificación y priorización de los intereses ambientales de las familias en situación de pobreza extrema del barrio El Ensueño, Córdoba, Quindío

\begin{tabular}{|c|c|c|c|c|l|}
\hline Carencia & Problema & Prioridad & $\begin{array}{c}\text { ¿Quiénes o qué } \\
\text { genera este } \\
\text { problema? }\end{array}$ & $\begin{array}{c}\text { ¿Por qué o cuáles son } \\
\text { las causas de este } \\
\text { problema? }\end{array}$ & Interés \\
\hline
\end{tabular}

Fuente: elaboración propia.

\section{Fase de análisis y discusión de resultados}

El investigador realiza el análisis de las teorías que ha recopilado a través de la revisión bibliográfica, acerca del objeto de estudio. El análisis (...) le permite reconocer vacíos, contradicciones, aportes y limitaciones de las teorías existentes en torno al tema que desea estudiar (Hurtado, 2000, p. $54)$.

Según Hurtado: "Las actividades que corresponden a esta fase son fundamentalmente reflexivas y analíticas en torno a la pregunta de investigación" (p. 54). En esta fase se llevaron a cabo las siguientes actividades:

\section{Categorización de la información}

Esta actividad implica establecer los criterios mediante los cuales se clasificará y agrupará la información obtenida a través del proceso de recolección de datos.

\section{Análisis}

Esta actividad implicó la comparación y relación de los datos obtenidos. Para el caso del material contenido en las entrevistas, implicó buscar relaciones entre contenidos o categorías, atribuirles un significado, integrarlos en un todo lógico y coherente de modo que esta fase converge a la discusión.

\section{Interpretación}

De acuerdo a Hurtado (2000, p. 186) esta actividad consiste en convertir las categorías encontradas en afirmaciones con significado, es decir, señalar el sentido de los resultados, qué significan las categorías y cuáles son las implicaciones a partir de los conceptos trabajados en el marco teórico. 


\section{Integración}

Esta actividad consiste en integrar los resultados obtenidos para cada objetivo de la investigación y convertirlos en un todo coherente, para dar respuesta a la pregunta de investigación y al objetivo general (Hurtado, 2000, p. 186).

\section{Explicación}

En esta actividad se toman los resultados del análisis, se les da significado de acuerdo al marco teórico, se "busca el sentido o la comprensión más amplia de los resultados y se compara con resultados de otras investigaciones" (Hurtado, 2000, p. 204). Se "Establece la conexión entre los datos y la teoría que se utilizó como marco de referencia" (p. 204).

\section{RESULTADOS Y DISCUSIÓN}

Las 6 familias en situación de pobreza extrema seleccionadas para el caso de estudio se relacionaron de una $u$ otra forma con las principales crisis que se presentaron durante la escala de tiempo utilizada: crisis económica, violencia, terremoto, demostrando que su vulnerabilidad, es decir sus limitadas capacidades para enfrentar situaciones de riesgo, hace que siempre lleven la peor parte cuando se presentan las crisis. Sumada a su vulnerabilidad se tiene la debilidad de las instituciones públicas y privadas para atender las necesidades de la población en situación de pobreza extrema, que no solo tiene que ver con temas presupuestales sino también con la forma como la oferta social del Estado es focalizada, atendiendo más la necesidad de mostrar cifras para lograr metas en un programa de gobierno o, lo que es aún peor, atendiendo a compromisos políticos adquiridos durante las campañas de los actuales gobernantes locales.

En ese orden de ideas, los intereses ambientales prioritarios de las familias en situación de pobreza extrema analizadas en el caso de estudio obedecen a carencias que se han profundizado con cada crisis, en las que la institucionalidad tanto pública como privada no ha sido efectiva para entregar su oferta social generando capacidades que permitan afrontar dichas situaciones como se explica en la Figura 2. 


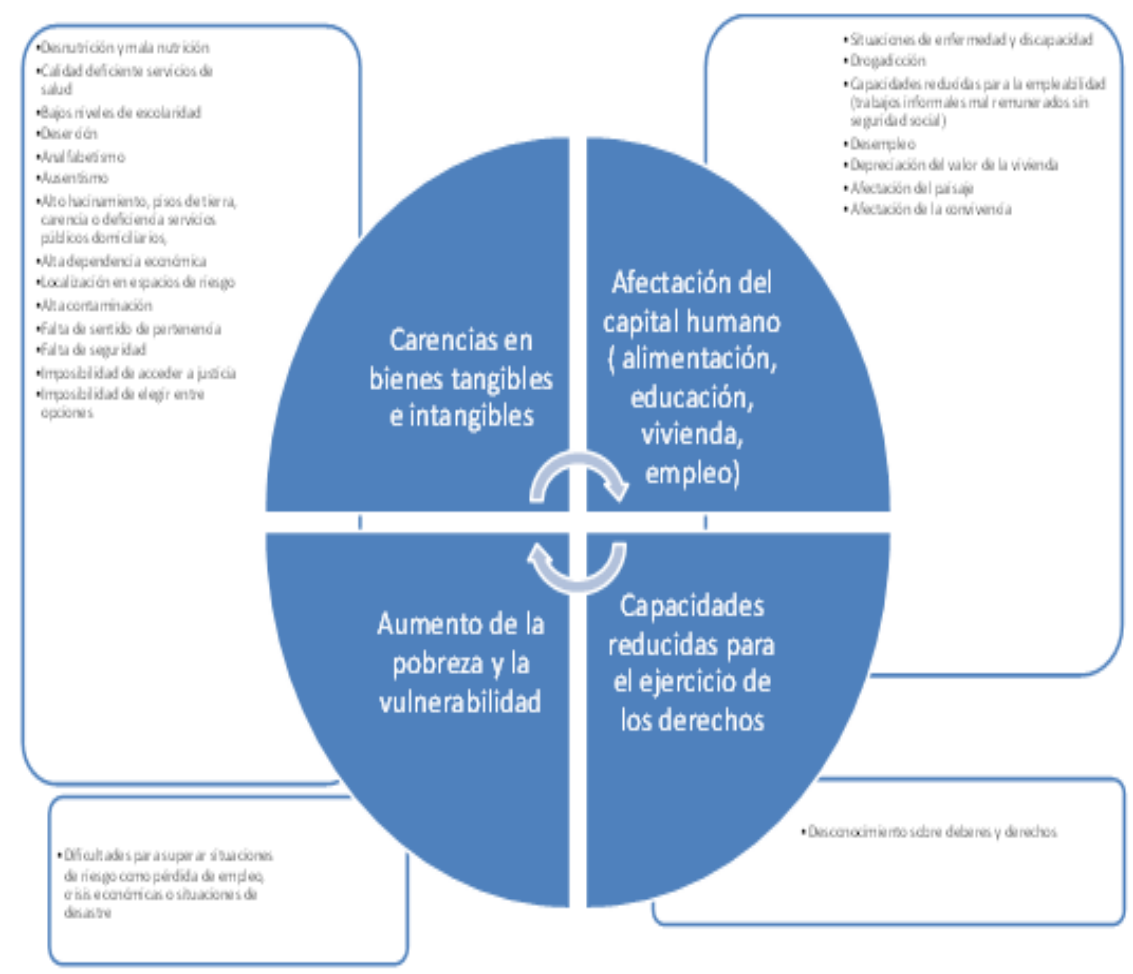

Fuente: elaboración propia.

Figura 2. Carencias y afectación de capacidades.

Del análisis de los intereses ambientales prioritarios de las familias en situación de pobreza extrema del barrio El Ensueño se encontró que, frente a la prioridad alta dada al riesgo de inundación y avalancha, se evidencia la preocupación de las familias por su vivienda, incluso más que por su propio bienestar pues todas manifestaron que sabían del riesgo del terreno donde estaban construyendo el barrio pero que, frente a la posibilidad de tener casa propia, no pensaron mucho en su seguridad.

El interés prioritario de las familias frente a la anterior carencia se identificó como la posibilidad de mitigar el riesgo a través de obras de ingeniería que permitan controlar las crecidas de las quebradas La Siberia y La Española. De igual forma, la implementación de programas preventivos para la limpieza del canal de la quebrada La Venenosa. La posibilidad de una reubicación solo es atractiva para el 
núcleo familiar número 3 que ha sido el más afectado por las inundaciones y el núcleo familiar número 5 que ha sido afectado por los deslizamientos del potrero.

Las familias, a pesar de ser conscientes del alto riesgo al que están expuestas, no consideran la posibilidad de una reubicación básicamente por temor a desmejorar las condiciones actuales de la vivienda que habitan, pues saben que las viviendas de interés prioritario tienen un área aproximada de $35 \mathrm{~m}^{2}$ frente a los $60 \mathrm{~m}^{2}$ de la vivienda del barrio El Ensueño.

De igual forma, manifiestan el amor por el barrio debido a que ellos mismos aportaron la mano de obra para su construcción, y su satisfacción con las viviendas que les entregaron, muchas argumentaron que era el mejor barrio del municipio porque las casas son muy amplias. Al respecto, Muñoz (2004 citado en Ontiveros, 2006, p. 2) plantea que la casa "además de ser un espacio físico, está hecha de identidades, relaciones y conflictos de quienes viven en su interior. La casa está marcada por los años y recuerdos que ella guarda".

Respecto a la prioridad alta dada a la creación de puestos de trabajo en el municipio, es apenas entendible que las familias en situación de pobreza extrema consideren que un empleo pueda mejorar su calidad de vida.

La generación de ingresos para un núcleo familiar es prioritaria en la medida en que se posibilita el acceso a bienes tangibles como la alimentación, la educación y la vivienda, los cuales permiten satisfacer bienes intangibles como el sentido de pertenencia, la autonomía, la justicia y la seguridad.

Se identificó, además, el interés por otro tipo de empleos que no estén relacionados con labores en el campo debido a lo mal remunerados y al esfuerzo físico que se requiere, haciendo evidente la necesidad de acceder a la educación superior, a la formación por competencias o a programas que promuevan el emprenderismo.

Por último, la prevención rehabilitación e inclusión de jóvenes con problemas de drogadicción hace parte de las prioridades de las familias, debido a las situaciones que se presentan tanto a nivel familiar como comunitario donde los jóvenes abandonan el sistema educativo convirtiéndose en una carga para sus padres y para la sociedad. Muchos de estos jóvenes se vinculan a grupos armados, bandas criminales o a la delincuencia común. 
Los padres se sienten impotentes frente a la situación de drogadicción de los hijos al no encontrar apoyo institucional, pues no existe oferta de servicios para estos jóvenes, ni siquiera el régimen subsidiado de salud cubre tratamientos de desintoxicación y rehabilitación. Es así como se toman medidas desesperadas como echar los jóvenes de los hogares, haciendo peor el problema pues sin el soporte económico de una familia terminan delinquiendo.

Una de las familias manifestaba, además, su preocupación por el ejemplo que estaban recibiendo los niños del barrio al ver a sus hermanos mayores o vecinos drogadictos, aumentando el riesgo de que el problema se incremente a futuro.

Frente a los intereses ambientales prioritarios de las instituciones públicas y privadas, que prestan sus servicios a las familias del caso de estudio, se encontró que desde la misión de cada una de estas entidades se tienen objetivos claros en el tema de generación de capacidades para la reducción de la pobreza extrema y de prioridad en atención a los más vulnerables, sin embargo, el alto nivel de centralismo en el cual los beneficiarios de los programas son seleccionados desde el nivel nacional obedeciendo más a criterios políticos que técnicos están llevando hacia la generación de conflictos, no solo entre las entidades y las familias directamente implicadas sino también entre las mismas entidades del Estado.

Este último hallazgo no se esperaba obtener, pues la investigación planteó la determinación de la existencia de conflictos entre los intereses ambientales prioritarios de las familias en situación de pobreza extrema y las entidades públicas y privadas, sin embargo se encontró que el Departamento Nacional de Planeación -DNP- y las Alcaldías no han conciliado los resultados de la metodología del SISBÉN versión III, existiendo reclamaciones de parte y parte, tanto en el diseño de la encuesta como en la aplicación de la misma. De otro lado, se encontró la disputa entre la Empresa Regional de Servicios Públicos del Quindío S.A. ESP NEPSA - y la Alcaldía de Córdoba por las competencias de cada entidad frente a la implementación del plan integral de residuos sólidos exigido por la autoridad ambiental.

Los conflictos entre las familias en situación de pobreza extrema y las entidades públicas y privadas están dados, principalmente, por la focalización de la oferta de entidades públicas como la Agencia Nacional para la Superación de la Pobreza Extrema -ANSPE-, el Departamento para la Prosperidad Social -DPS- y el Servicio Nacional de Aprendizaje -SENA- que obedecen a la prioridad de mostrar cifras de superación de pobreza extrema desconociendo la vulnerabilidad de las 
familias más necesitadas, generando aun más desigualdad. De otro lado, los conflictos de uso del suelo y de información que se presentan frente a la Reserva Forestal Central donde está localizado más del 90\% del municipio de Córdoba, impidiendo a los potenciales beneficiarios del programa de subsidio de tierras del Instituto Colombiano de Desarrollo Rural -INCODER- participar de dicha oferta. En la Figura 3se presentan los agentes y conflictos identificados durante la investigación.

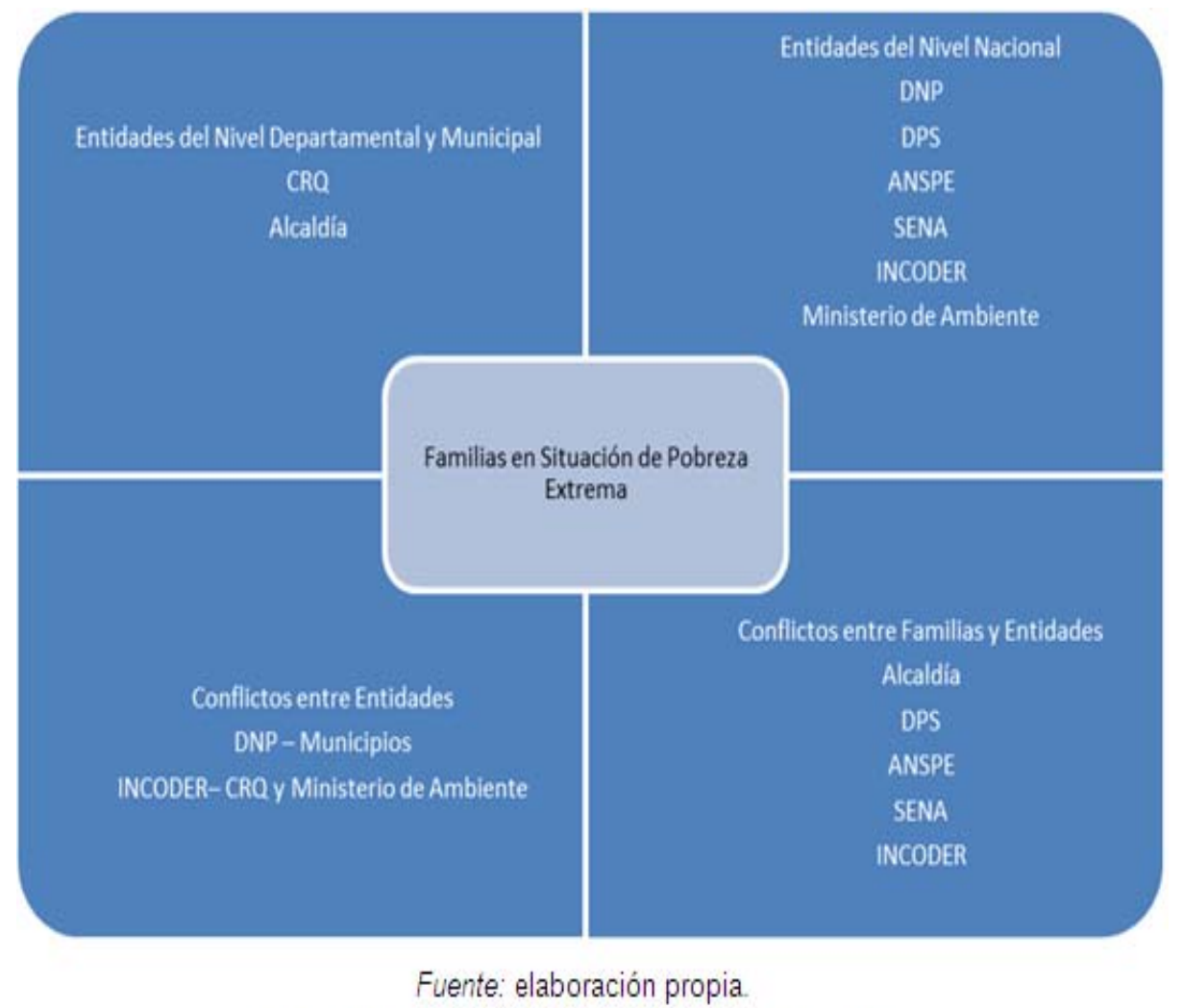

Figura 3. Agentes y conflictos identificados.

Para terminar, los resultados de la investigación evidencian el desconocimiento de las entidades públicas y privadas frente a la pobreza, los imaginarios reales de las personas en esta situación, la manera en que se las arreglan para sobrevivir y reproducirse adaptándose a una sociedad cada vez más competitiva y excluyente. 


\section{CONCLUSIONES Y RECOMENDACIONES}

Son diversos los factores que condicionan el nivel de exposición de las familias a las crisis y su capacidad de hacerles frente: el tipo de crisis, la condición socioeconómica, el capital social, las redes de apoyo y la oferta social del Estado. Las carencias en el bien tangible del ambiente afectan negativamente las capacidades de las personas en la medida en que sus manifestaciones causan daño a la salud, los medios de vida, la seguridad e incluso el valor del suelo o de la propiedad perpetuando y profundizando la situación de pobreza. Esto también significa que las inversiones que se hagan para suplir estas carencias podrían generar condiciones para ampliar capacidades de órdenes superiores o más complejas.

La imagen de las entidades públicas, principalmente de las del nivel nacional y el cumplimiento de sus metas implica, como en el caso de la ANSPE, invertir las prioridades focalizando la oferta del Estado para beneficiar a personas o familias cuya situación socioeconómica puede estar mejor en comparación con otras. De igual manera, esta forma de priorizar la oferta aumenta la desigualdad entre las regiones, incluso entre los mismos municipios de un departamento como es el caso del Quindío.

Además de existir trampas de pobreza extrema también existen las trampas del desarrollo, las cuales impiden a los más pobres acceder a oportunidades para la educación y el trabajo. Estas trampas del desarrollo tienen que ver directamente con las políticas de competitividad que implementan las instituciones públicas, como el SENA que no puede ofertar formación titulada en el municipio de Córdoba por tener todos sus programas acreditados como de "alta calidad". Esta situación incrementa la desigualdad, ya que las familias del barrio El Ensueño no tienen recursos para que los jóvenes se desplacen hacia la ciudad de Armenia para adelantar estudios de formación técnica y tecnológica, teniendo como única opción el acceso a cursos cortos que no están mejorando las capacidades ni competencias de las personas para acceder a empleos diferentes a los disponibles en el sector agropecuario.

La centralización de la focalización de la oferta social del Estado impide que los municipios y departamentos, que son los que conocen los problemas sociales y ambientales, participen de la toma de decisiones frente a la población que debería beneficiarse, generando conflictos entre los distintos niveles de las entidades públicas. El caso más concreto es el del DPS, en el que la focalización de los 
programas se realiza desde el nivel central obedeciendo a las prioridades de la Red Unidos en cuanto a promoción de familias. Este sistema de promoción favorece la concentración de la oferta social del Estado en municipios donde las familias presentan mejores indicadores de calidad de vida y están más cerca de cumplir con las condiciones diseñadas para ser declaradas no pobres o pobres moderadas. Es el caso de los municipios de cordillera en el departamento del Quindío, entre ellos Córdoba, donde la oferta del DPS se limita a unos pocos programas, mientras que para los municipios de la zona plana del departamento la oferta es mucho más amplia.

Los conflictos ambientales, además de sus implicaciones para la conservación y la sostenibilidad ambiental, envuelven objetivos de desarrollo económico y social para la población afectada, especialmente para la más pobre. El área de Reserva Forestal Central declarada en 1959 por la Ley Segunda y que afecta a todos los municipios del Quindío localizados en la cordillera Central, entre ellos específicamente a Córdoba, y que en cierta medida ha impedido que grandes proyectos mineros se lleven a cabo en esta zona, también ha impedido que el INCODER adjudique terrenos para proyectos productivos que permitan una mejor calidad de vida para las familias vulnerables.

Los conflictos ambientales en un país tan diverso natural y culturalmente como Colombia plantean desafíos a la comunidad académica para su abordaje y estudio, desde los marcos teóricos y las metodologías hasta la presentación de los resultados.

Aunque existe una herramienta para solicitar ante el Ministerio de Ambiente la sustracción de áreas de la Reserva Forestal Central, ni la población, ni las entidades públicas del departamento del Quindío o del municipio de Córdoba han adelantado dicha gestión. Lo anterior evidencia, para el caso de la población, la falta de información y de acompañamiento por parte de entidades como el INCODER o la Red Unidos. De otro lado, las entidades se excusan en la posibilidad de que la minería a gran escala pueda sacar ventaja de estas sustracciones para legalizar operaciones, sin embargo, la información no es suficientemente clara entre los funcionarios y parece más bien puras especulaciones que reflejan la debilidad institucional del departamento del Quindío y la falta de experticia de los funcionarios que laboran en estas entidades.

Los intereses ambientales prioritarios pueden ser un instrumento desde las ciencias ambientales para aportar a la evaluación de las políticas públicas en 
Colombia, ya que permiten unificar variables económicas, sociales y ambientales a través de la visión integral de una problemática ambiental. De igual forma, en el escenario de implementación de un posible acuerdo de paz con la guerrilla de las FARC, en el que se prevé la necesidad de diseñar e implementar políticas públicas en construcción de paz para conciliar los conflictos sociales y ambientales de los territorios más golpeados por el conflicto armado, abordar los intereses ambientales prioritarios permitiría la identificación y triangulación de información social y ambiental para la resolución de estos.

\section{POTENCIAL CONFLICTO DE INTERESES}

No hay conflicto de intereses.

\section{FUENTES DE FINANCIACIÓN}

Recursos propios.

\section{REFERENCIAS}

- Bonilla-Castro, E., \& Rodríguez Sehk, P. (1997). Más allá del dilema de los métodos. La investigación en ciencias sociales. Grupo Editorial Norma.

- Corredor, C. (1998). El problema de la pobreza: concepto, mediciones, instituciones y políticas. Ponencia dictada en Seminario Internacional sobre Pobreza. Colciencias-CINEP, Bogotá.

- Cox, R. (1996). El saber local, metodologías y técnicas participativas. La Paz: NOGUB-COSUDE/ CAF.

- Díaz, C. (2007). Metodología interdisciplinaria desde el estudio de la problemática ambiental del tramo urbano de la cuenca del río Consota: hacia el fortalecimiento de la gestión ambiental local. (Tesis de magíster). Universidad Nacional de Colombia, Manizales. 
- Fontaine, G. (2004). Enfoques conceptuales y metodológicos para una sociología de los conflictos ambientales. En M. Cárdenas, M. Rodríguez (Eds.). Guerra, sociedad y medio ambiente (pp. 503-533). Bogotá: Foro Nacional Ambiental.

- Gudynas, E., \& Evia, G. (1991). La Praxis por la Vida - Introducción a las metodologías de la Ecología Social. Montevideo: CIPFE - CLAES NORDAN.

- Hurtado, J. (2000). Metodología de la investigación holística. Caracas: Fundación Sypal.

- Muñoz, O. (2004). Urbanizaciones piratas. Bogotá: Ediciones Doctrina y Ley Ltda.

- Ontiveros, T. (2006). Vivienda popular urbana y vida cotidiana. Recuperado de http://encontrarte.aporrea.org/media/41/vivienda \%20popular\%20y\%20vida\%20cotidianapara.pdf

- $\quad$ PNUD -Programa de Naciones Unidas para el Desarrollo-. (2011). Informe Sobre Desarrollo Humano 2011. Sostenibilidad y Equidad: Un mejor futuro para todos. Washington.

- Posada, A. (2009). Intereses ambientales: reflexiones sobre la aplicación de lo difuso.Revista Ingenierías, 8(4), 11-20.

- UNIFEM -Fondo de Desarrollo de las Naciones Unidas para la Mujer-. (2010). Estudio sobre tolerancia social e institucional a la violencia basada en género en Colombia. Bogotá.

1. Magíster en Ciencias Ambientales. Universidad Tecnológica de Pereira. Pereira, Colombia.paoandrea1982@hotmail.com. ORCID: 0000-0003-3120-4201 
Para citar este artículo: López-Arbeláez, P.A. (2016). Intereses ambientales prioritarios de la población en situación de pobreza extrema. Estudio de caso: barrio El Ensueño, Córdoba, Quindío. Revista Luna Azul, 43, 229-250. Recuperado de http://200.21.104.25/lunazul/index.php?option=com_content \&view=article\&id=200

Esta obra está bajo una Licencia de Creative Commons Reconocimiento CC BY

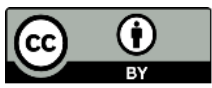

